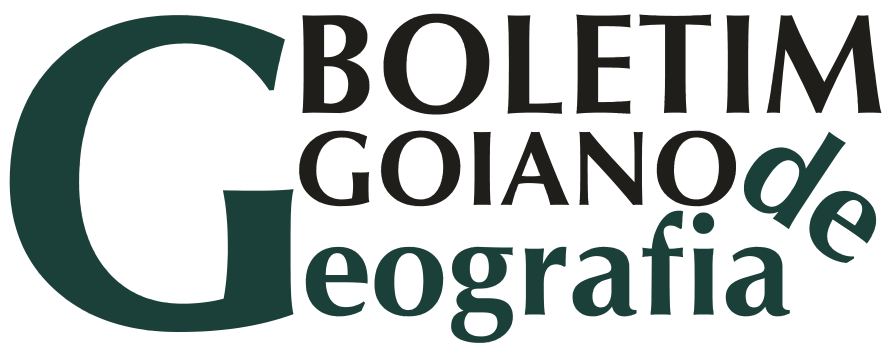

INSTITUTO DE ESTUDOS

SÓCIO-AMBIENTAIS/GEOGRAFIA

VOL. 23 - No 1 - JAN./ JUN. 2003

$\infty$
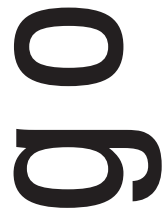

$\square$
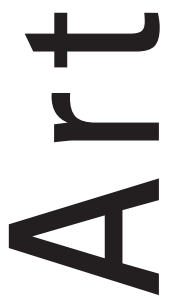


\title{
LE CAMEROUN: UN PAYS AUX POTENTIALITES TOURISTIQUES SOUS EXPLOITEES
}

\author{
Mesmin Tchindjang* \\ Kengne Fodouop**
}

\section{Résumé}

Avec une large extension en latitude $\left(11^{\circ}\right.$, Fig.1a), un relief varié constitué de plaines, de plateaux et de montagnes, un climat et une végétation diversifiés ainsi qu'un peuplement humain hétérogène, le Cameroun apparaît comme une terre de tourismes par excellence. Il est reconnu par des experts comme une Afrique en miniature. Cependant, jusqu'à présent, le pays n'a pas mis à profit ce riche potentiel naturel. En effet, la contribution de l'activité touristique au PIB du Cameroun est très faible (à peine 1\%). Le manque d'outils statistiques fiables et l'absence de politique d'incitation rendent difficiles toute entreprise d'évaluation des ressources et des potentialités touristiques du pays. A vrai dire, l'activité touristique du Cameroun est en déclin comme en témoignent les rares statistiques sur les fréquentations et recettes y afférentes.

Mots clés: Afrique en miniature/ Culture/Fréquentations touristiques/ Tourisme.

\section{Introduction}

L'évolution des comportements dans le monde pousse de plus en plus de nombreux touristes à la recherche des destinations vierges. L'Afrique en est une avec un record de 29,5 millions de touristes en 1999 (27M en 1998) avec un accroissement de plus de 9\%. Mais elle montre beaucoup de disparités régionales (Fig.1b). Cependant le Cameroun ne bénéficie pas de ces avantages et de cette croissance. L'activité touristique camerounaise date des lendemains de l'Indépendance. Elle fut prospère. Cette période de prospérité a connu son apogée en 1978, année seuil où le total des fréquentations et surtout celui

\footnotetext{
* Université de Yaoundé I, Laboratoire de Géomorphologie, Département de Géographie, Cameroun. E-mail: mtchind@yahoo.fr

**Université de Yaoundé I, Laboratoire de Géographie humaine, Département de Géographie. E-mail: fkengne@uycdc.uninet.cm
} 
TCHINDJANG, M.; FODOUOP, K. Le Cameroun: un pays aux potentialites touristiques sous exploitees.

des recettes touristiques a atteint un niveau jamais égalé de nos jours en valeur absolue avec un taux d'accroissement de $23 \%$. Onze ans après, l'organisation administrative des structures de gestion touristique a connu un développement optimal avec la création du MINTOUR en 1989. Cependant il est fort curieux de constater que depuis cette date, le tourisme au lieu d'aller de l'avant connaît des difficultés considérables avec la dégradation des structures d'accueil et le manque de plan directeur ou de planification. De nos jours, le tourisme se cantonne exclusivement à certaines Provinces (Littoral et Centre avec 90\% de la clientèle plus de $50 \%$ des infrastructures et $70 \%$ de recettes. Les sites les plus fréquentés sont de vieux sites connus. Pourtant, il en existe un peu partout. En effet, de la forêt équatoriale à la savane et aux steppes septentrionales en passant par les hautes terres de l'Ouest et de l'Adamaoua, le Cameroun dispose d'un géopotentiel touristique exceptionnel et favorable avec de puissants facteurs d'attraits jusque là insoupçonnés. Cette contribution veut dégager les carences en s'appuyant sur les quelques données statistiques existant sur le plan national et proposer quelques solutions aux difficultés relevées.

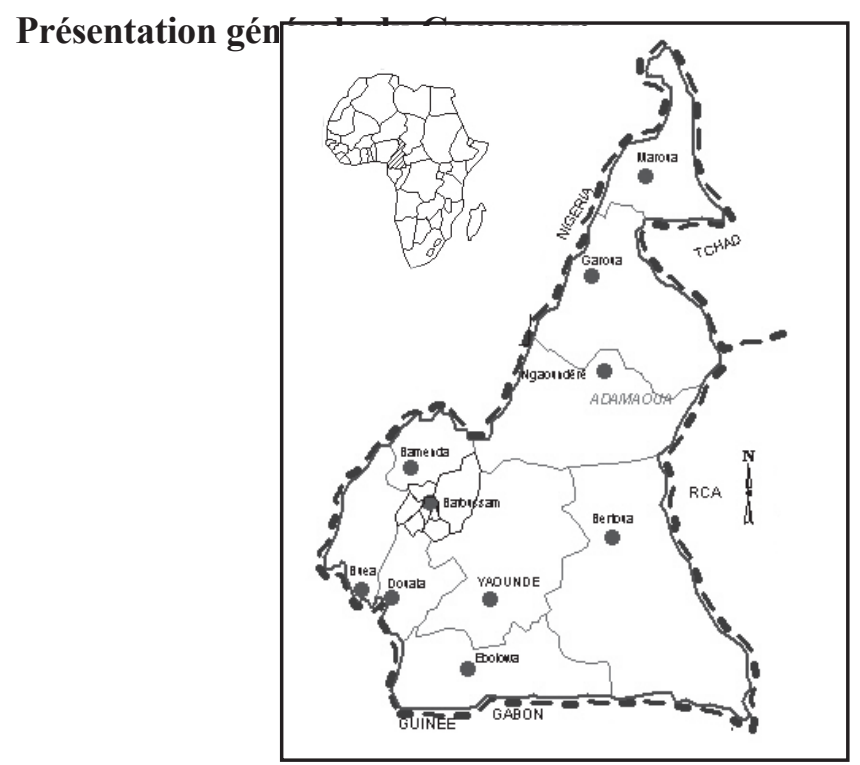

Fig.1a - Carte de localisation 
TCHINDJANG, M.; FODOUOP, K. Le Cameroun: un pays aux potentialites touristiques sous exploitees.

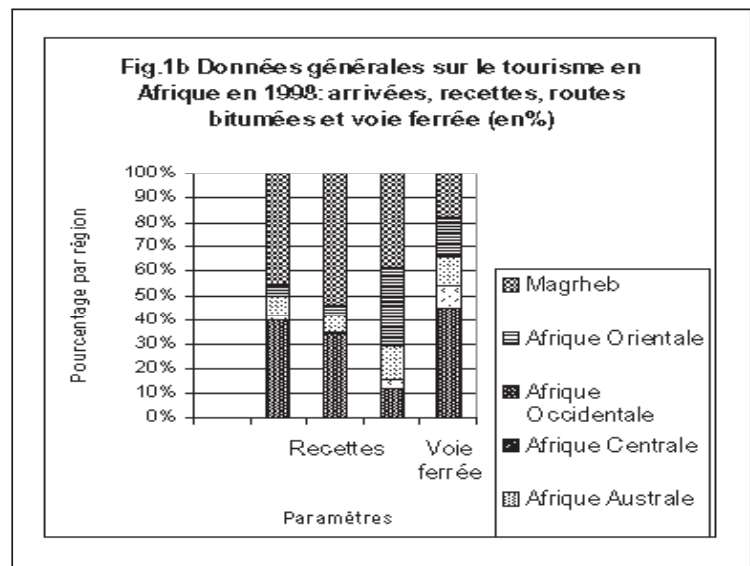

Fig.1b-Données générales sur le torisme en Afrique en 1998: arrivés, recettes, routes bitumées et voie ferrée (en \%)

Le Cameroun s'étire sur plus de 1200 km du lac Tchad au Golfe de Guinée entre 2 et $13^{\circ}$ de latitude N (Fig.1a). Cette large extension en latitude lui vaut de bénéficier de plusieurs types de reliefs qui s'échelonne des hautes terres (point culminant au Mont Cameroun, $4.095 \mathrm{~m}$ ) aux régions basses (plaine côtière et bassin du lac Tchad, $300 \mathrm{~m}$ ). Le Cameroun est donc constitué d'une mosaïque de relief dont les découpages morphologiques de l'histoire fait apparaître des lithômes (surfaces rocheuses susceptibles d'engendrer le tourisme telles que les monolithes); les hydrômes (surfaces d'eau propices au tourisme: source, chute, lacs, fronts de mer) les phytômes (végétation susceptibles de consommation touristique).

La configuration topographique confère à ce pays une variété de climats et de végétation dont 5 grands types sont reconnus du Sud au Nord :

- Un climat équatorial et subéquatorial $(1.500-2.000 \mathrm{~mm})$ qui donne lieu à une luxuriante forêt dense équatoriale. La forêt camerounaise fait partie de la grande forêt du Bassin du Congo qui est après le Bassin amazonien au Brésil, la seconde région forestière tropicale du Globe.

- Un climat de mousson côtier avec des précipitations abondantes 3.000-4.000 mm sur le littoral de Kribi et de Douala. Ces abondantes précipitations donnent lieu à une forêt littorale et des mangroves dans les estuaires. 

exploitees.

- Un climat montagnard lié à la mousson qui frappe de plein fouet l'Ouest du pays y déversant des précipitations abondantes (2.000-3.000 $\mathrm{mm}$ ) parfois exacerbées par le relief. L'orientation du trait de côte dans la région de Debunscha, située sur le flanc occidental du mont Cameroun $(4.100 \mathrm{~m})$ donne lieu à des trombes qui font plus de $10.000 \mathrm{~mm}$ d'eau par an. Cette station de Debunscha devient de ce fait la seconde station la plus arrosée du monde après Cherrapunji en Inde.

- Un climat soudanien d'altitude $(1.500 \mathrm{~mm})$ et un climat soudanien $(1.000$ $\mathrm{mm}$ ) à mesure que l'on remonte vers le Nord.

- Un climat sahélien dans l'Extrême Nord du Cameroun (600 mm)

Le Cameroun par sa disposition naturelle et les faveurs du climat dispose d'au moins 120 sites touristiques recensés et répartis dans l'ensemble du triangle national avec une vingtaine d'aires protégées dont au moins une par province. Par ailleurs, le pays avec ses 15 millions d'habitants compte plus de 200 ethnies constituant autant d'atouts et d'attraits culturels pour le tourisme.

\section{Quelques caractéristiques du Cameroun}

\section{A - Outils statistiques déficients}

Considéré à juste titre par les spécialistes comme une Afrique en miniature, ce pays au peuplement humain hétérogène apparaît comme une terre de tourismes par excellence en Afrique comparativement au Sénégal, Kenya etc. où il n'existe pas autant de diversités. En effet, ce géopotentiel touristique exceptionnel, puissant facteur d'attrait et de diversification touristique, n'a pas donné naissance à un tourisme national et international de qualité à la dimension des attentes. Aussi, les quelques statistiques disponibles nous montrent que sur les 120 sites touristiques recensés, seuls $20 \%$ sont exploités actuellement pour le tourisme international. Pourtant, depuis la création du MINTOUR en 1989 (Ministère du Tourisme), l'OMT attendait au Cameroun 500.000 touristes en l'an 2000. Il n'en est rien, le Cameroun a enregistré moins de 150.000 touristes pour 40 millions de dollars US. Le tableau I montre bien que sur 39 pays d'Afrique, le Cameroun occupe la $24^{\mathrm{ème}}$ place. En comparant le Cameroun au Sénégal qui n'atteint pas la barre des 500.000 touristes, les caractéristiques suivantes peuvent être relevées pour l'année 1998. 


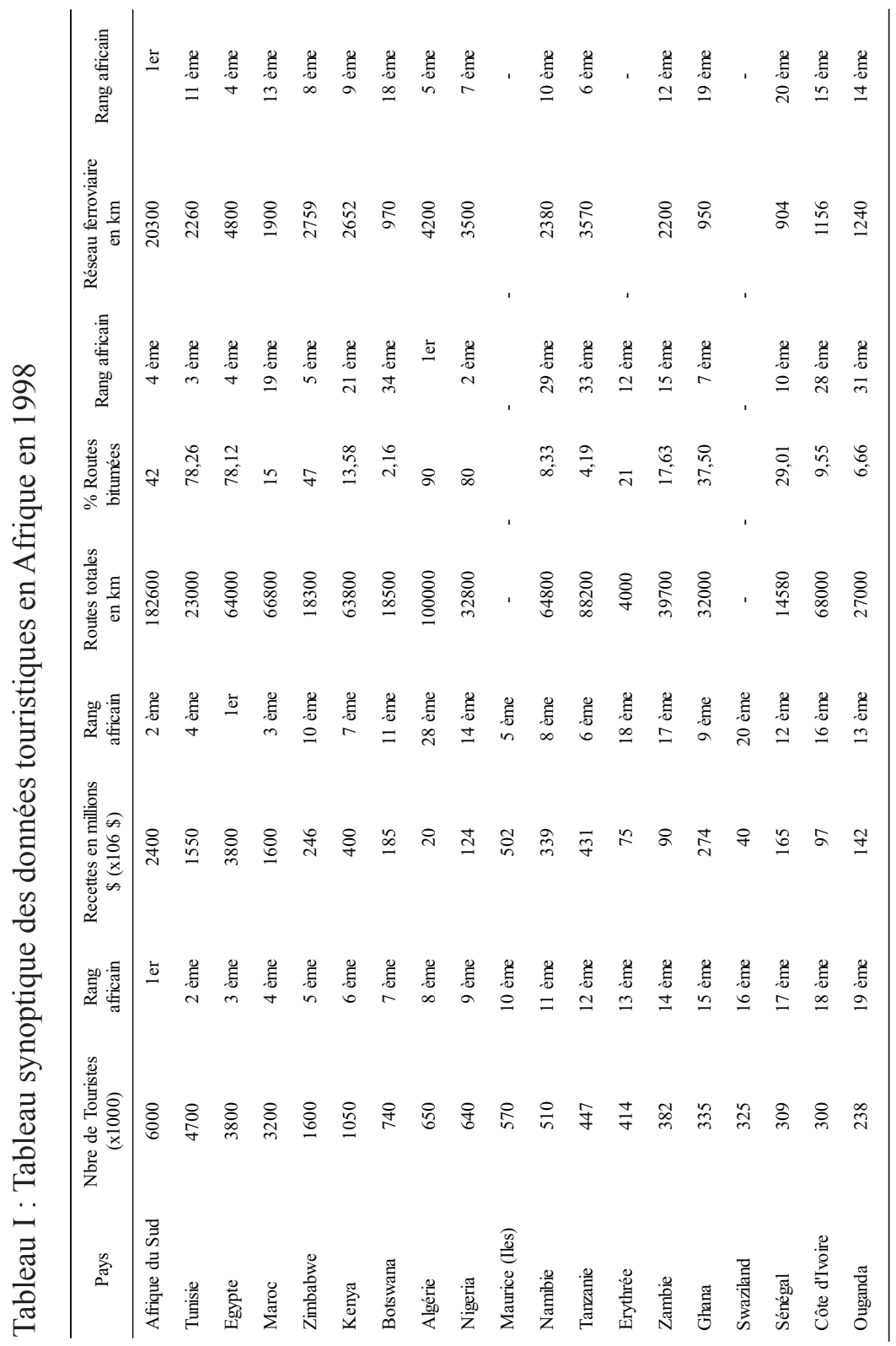



exploitees.

言

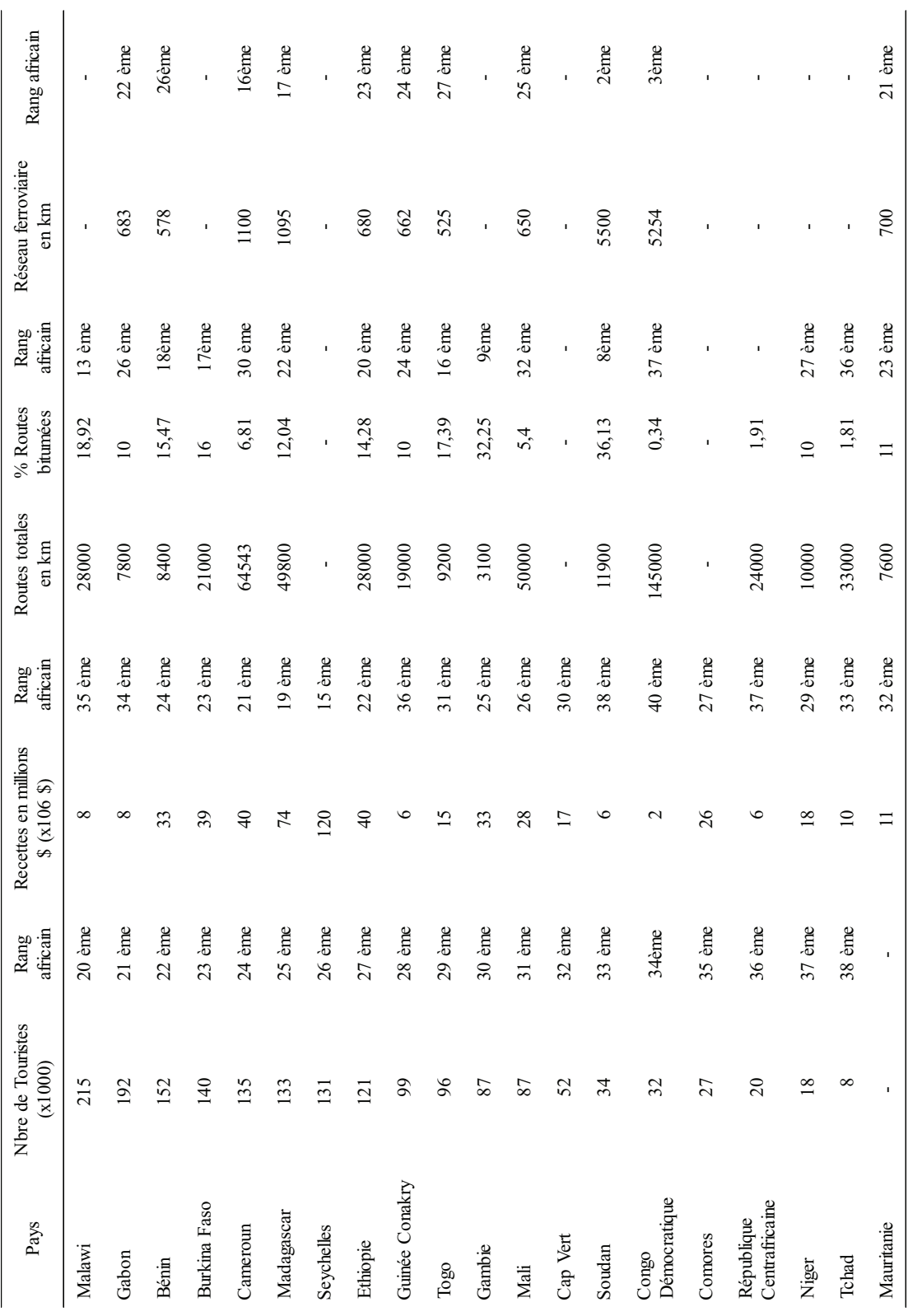


TCHINDJANG, M.; FODOUOP, K. Le Cameroun: un pays aux potentialites touristiques sous exploitees.

CAmeroun Senegal

Offre touristique

Nombre total

d'Etablissements: $\quad 188$

Nombre total de chambre: $\quad 7.136 \quad 8239$

Nombre total de lits: $\quad 15.000$

Nombres d'Aires Protégées: $\quad 20 \quad 10$

Demande touristique

Nombre de touristes

Non-Résidents

135.000

352.389

Nuitées des N. Résidents:

283.500

1.449 .358

Recettes:

40 MS US

165MS US

Performances

Taux d'occupation:

$15-17 \%$

$40 \%$

Durée moyenne de Séjour:

$1,85-2$

$3,9-4$

Dans ces caractéristiques il faut ajouter un développement timide du tourisme jusqu'en 1989 caractérisé par un certain désordre dans le domaine du classement des établissements. Ainsi, on compte près de 188 hôtels classés avec 301 établissements non classés au Cameroun. La balance touristique est négative et la contribution du Tourisme au PIB est inférieure à $1 \%$ alors qu'au Sénégal, le tourisme représente la $2^{\text {ème }}$ source de devises. Pourtant, le Cameroun dispose d'un potentiel hôtelier suffisant (Fig. 2 a, b et c), mais leur taux de fonction touristique demeure faible. Par ailleurs, le développement de la formation professionnelle est faible en matière touristique au Cameroun, il en est de même avec la promotion touristique en association avec le secteur privé. L'appareil statistique est également faible. Certes, l'on a mis en place une direction des statistiques mais, cet outil est déficient comme le montrent les chiffres et les graphiques sur l'évolution du potentiel hôtelier qui montre (Fig. $3 \mathrm{a}, \mathrm{b}$ et $\mathrm{c}$ ) des périodes creuses. En réalité les statistiques de la fréquentation touristique ne sont disponibles parfois qu'après 3 à 5 ans. Dans ce sens leur mise à jour devient difficile et peut comporter beaucoup d'erreurs. 
TCHINDJANG, M.; FODOUOP, K. Le Cameroun: un pays aux potentialites touristiques sous exploitees.

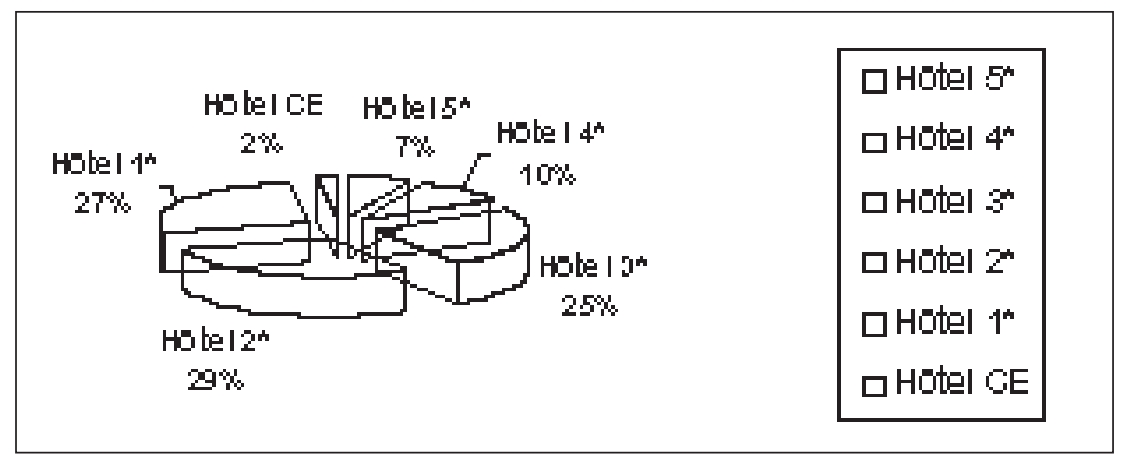

Fig.2a - Classification des etablissemnts d'hébergements au Cameroun

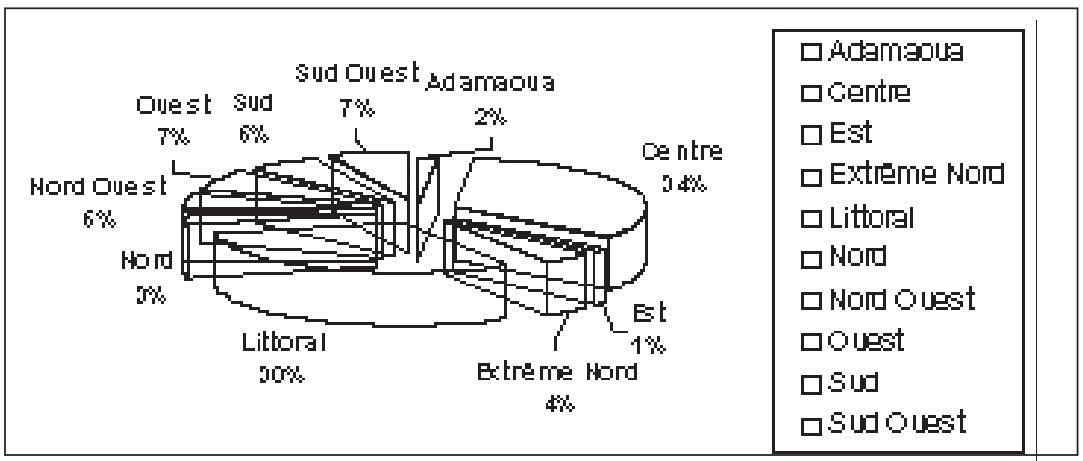

Fig.2b - Répartition du potentiel hôtelier par province au Cameroun (nbre de lits)

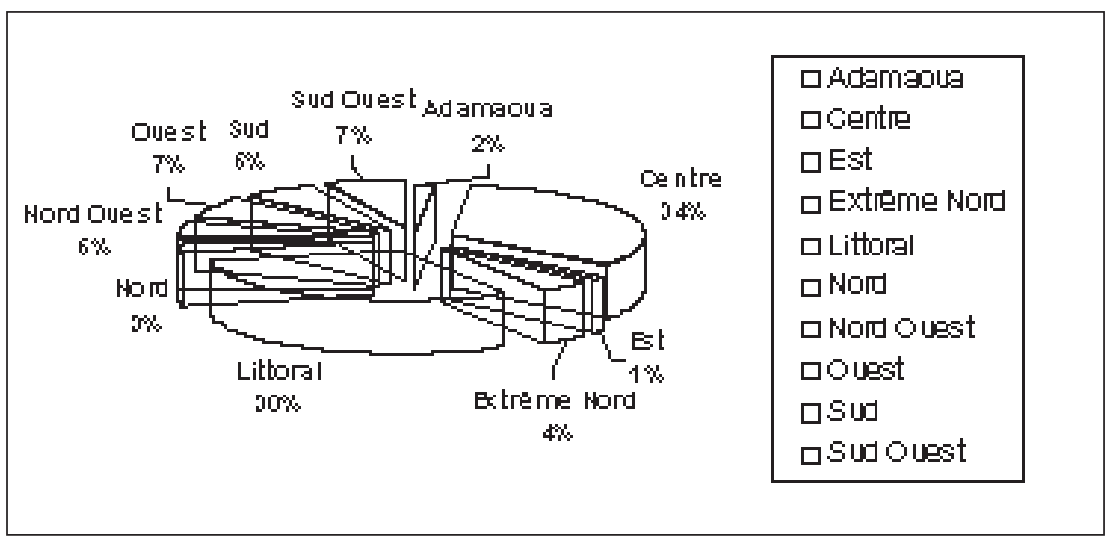

Fig.2c - Répartition du potentiel hôtelier par province au Cameroun (nbre de cham- 
TCHINDJANG, M.; FODOUOP, K. Le Cameroun: un pays aux potentialites touristiques sous exploitees.

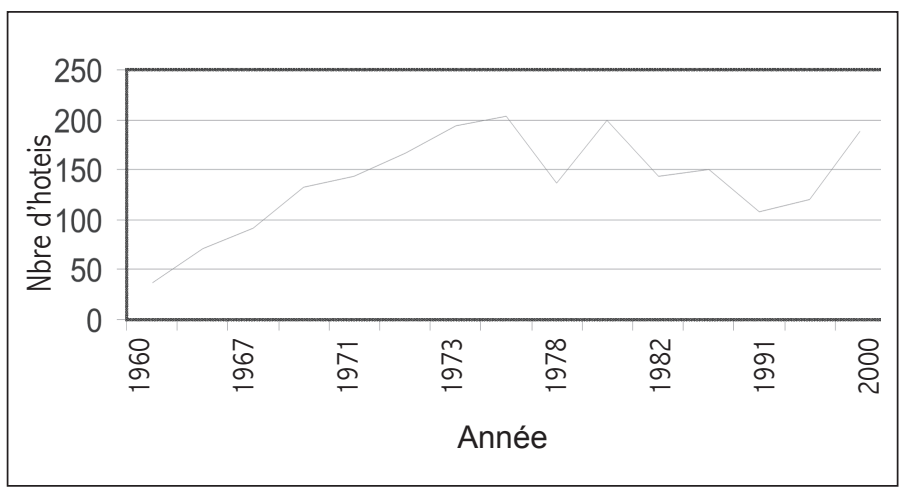

Fig.3a - Evolution du potentiel hôtelier du Cameroun (1960-2000)

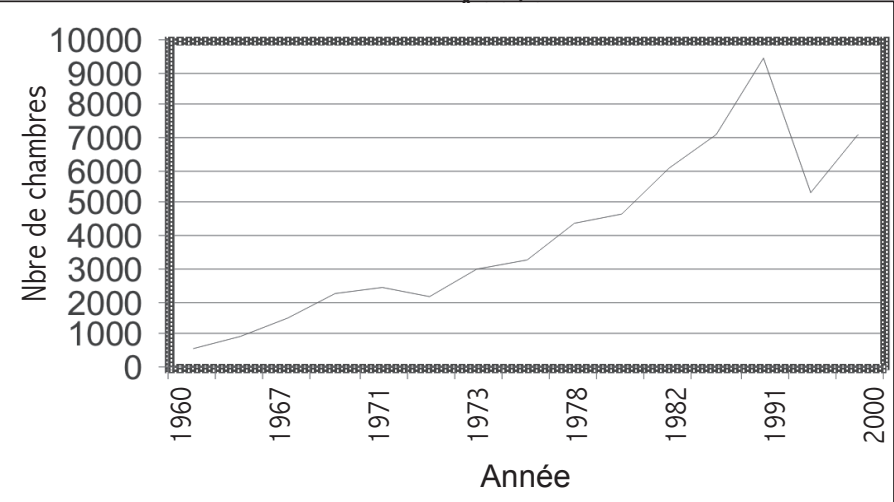

Fig. $3 b$ - Evolution du potentiel hôtelier au Cameroun (1960-2000)

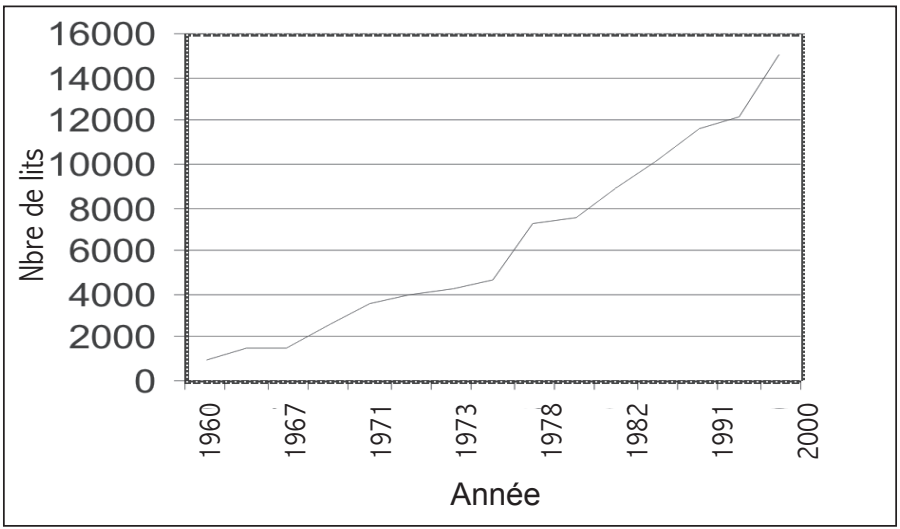

Fig.3c - Evolution du potentiel hôtelier du Cameroun (1960-2000) 
10

TCHINDJANG, M.; FODOUOP, K. Le Cameroun: un pays aux potentialites touristiques sous exploitees.

Néanmoins les statistiques les plus récentes montrent que l'activité touristique est en déclin au Cameroun. En prenant la période récente de 1976 à 1995, les données montrent une baisse progressive de la fréquentation touristique avec une moyenne de 386.751 arrivées pour 274.486 Résidents et 112.265 non-Résidents. L'ensemble donne respectivement 671.131, 438.999 et 233.132 Nuitées. Ce qui apparaît insignifiant (Fig. 4 a et b ). Dans tous les cas, l'évolution touristique au Cameroun est régressive et les statistiques sont parfois bancales et inexactes.

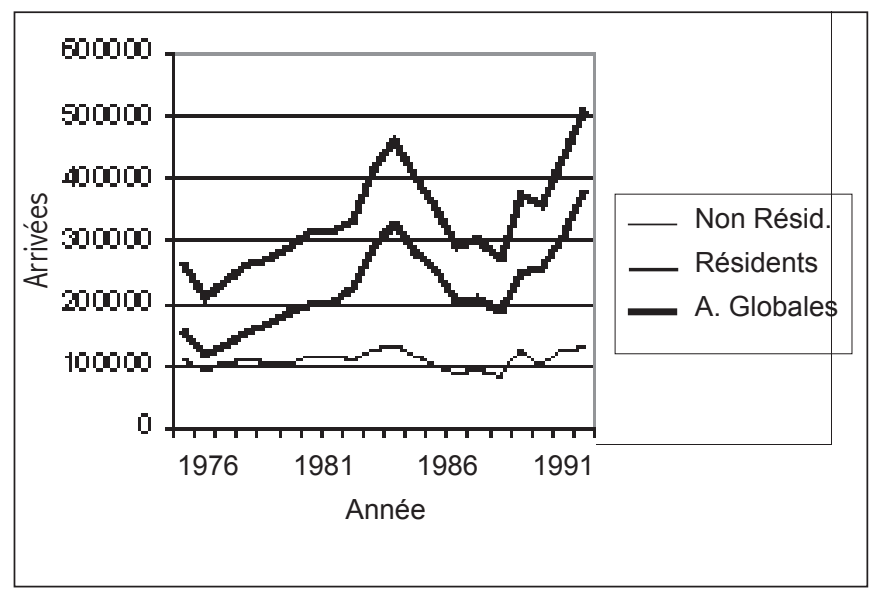

Fig.4a - Arrivées dans les etablisssements d'hébergement (1976-1995)

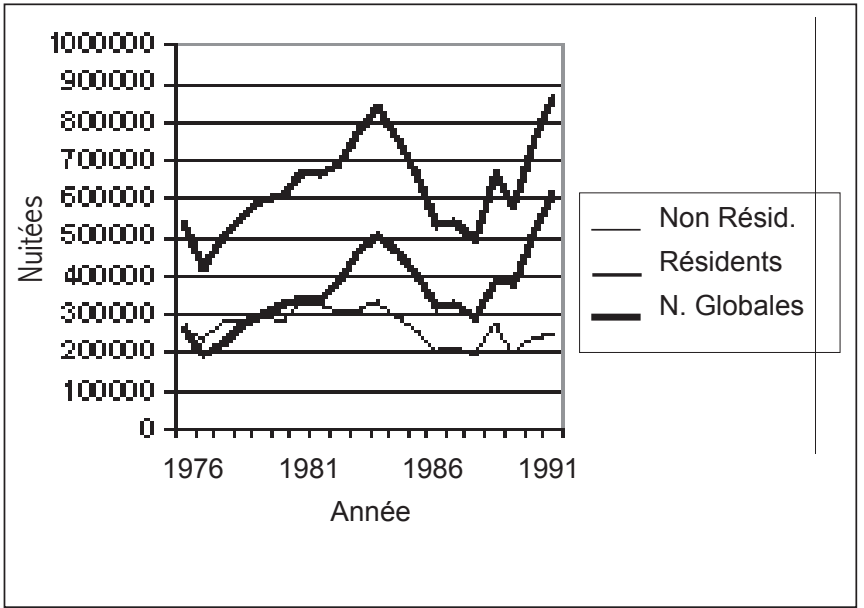

Fig. $4 b$ - Nuitées dans les etablissements d'hébergement (1976-1995) 
TCHINDJANG, M.; FODOUOP, K. Le Cameroun: un pays aux potentialites touristiques sous exploitees.

\section{B - Des disparités provinciales des fréquentations et recettes touris- tiques}

L'évolution de la fréquentation touristique ainsi que celle des recettes touristiques par province montre de grandes disparités. Le Littoral avec Douala, ancienne cité balnéaire du Cameroun apparaît comme la province la plus visitée du pays. Elle englobe à elle seule 35 à $40 \%$ des Arrivées et Nuitées et $55 \%$ des recettes avec une durée moyenne de 2, 3 jours et un taux d'occupation supérieur à $20 \%$. On constate que la plupart des visiteurs étrangers du Cameroun demeurent dans cette ville pourtant le Grand Nord détenteur de 4 parcs nationaux traîne la patte.

Yaoundé, capitale politique, ville des Congrès met la Province du Centre à la seconde place pour ce qui est de la clientèle étrangère, du volume des recettes $(27 \%)$ et du taux d'occupation (25\%). En général les autres provinces y compris celle du Nord et de l'Extrême Nord réputées pour leurs Parcs Nationaux font piètre figure dans le domaine du tourisme et jouent par conséquent un rôle très secondaire. Dans tous les cas, les recettes touristiques sont en baisse, même si la courbe indiciaire semble montrer une augmentation, celle des variations montre

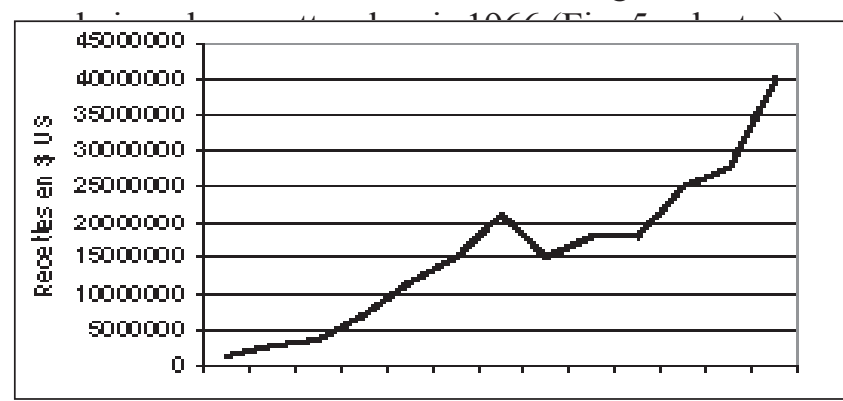

Fig.5a - Evolution des recettes touristiques au Cameroun (1975-1998)

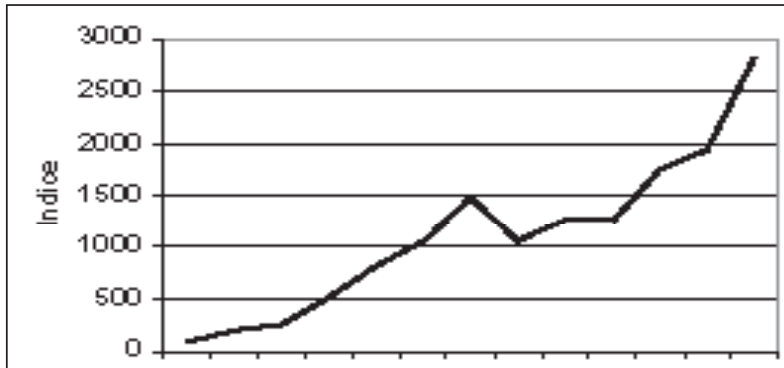

Fig. $5 b$ - Evolution indiciaire des recettes touristiques du Cameroun (1975-1998) 
TCHINDJANG, M.; FODOUOP, K. Le Cameroun: un pays aux potentialites touristiques sous exploitees.

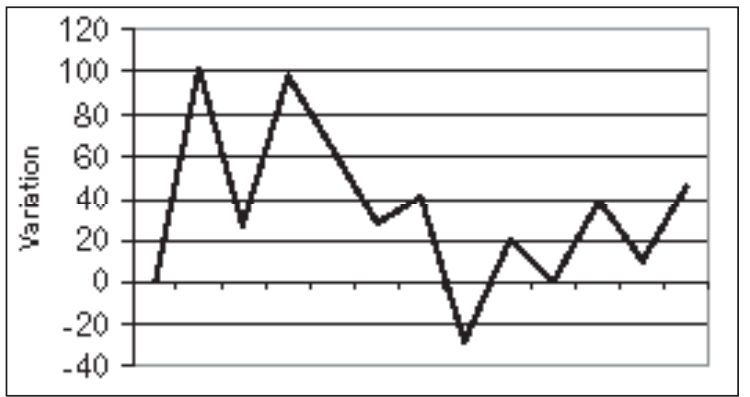

Fig.5c - Variation des recettes touristiques au Cameroun (19751998)

\section{C - Bilan global}

Dans l'ensemble donc, le Cameroun atteint difficilement 600.000 nuitées globales et la proportion des Non résidents est en baisse en dépit d'une certaine diversification de la clientèle étrangère dominée à plus de $40 \%$ par les Français cuivic des $\Delta$ frisaine (Fir $\mathrm{h}$ a of h I I a haicce des nuitées s'accompagne d'un

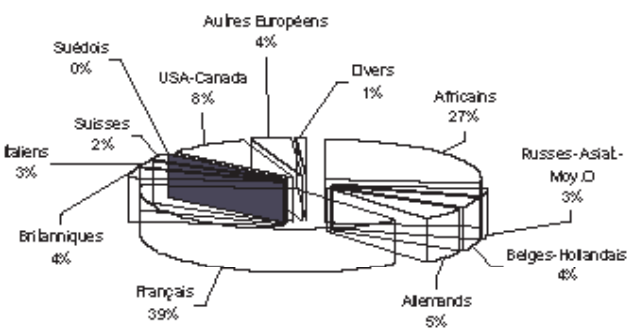

\begin{tabular}{|c|c|}
\hline 口 Russes-Asat-koy D & 口 Bejges-Holandais \\
\hline a franças & 口Britarniques \\
\hline 口 Suisses & asuedois \\
\hline 口 Autres Buropeens & achers \\
\hline
\end{tabular}

Fig.6a - Arrivées de touristes par nationalité au Cameroun (1976-1995)

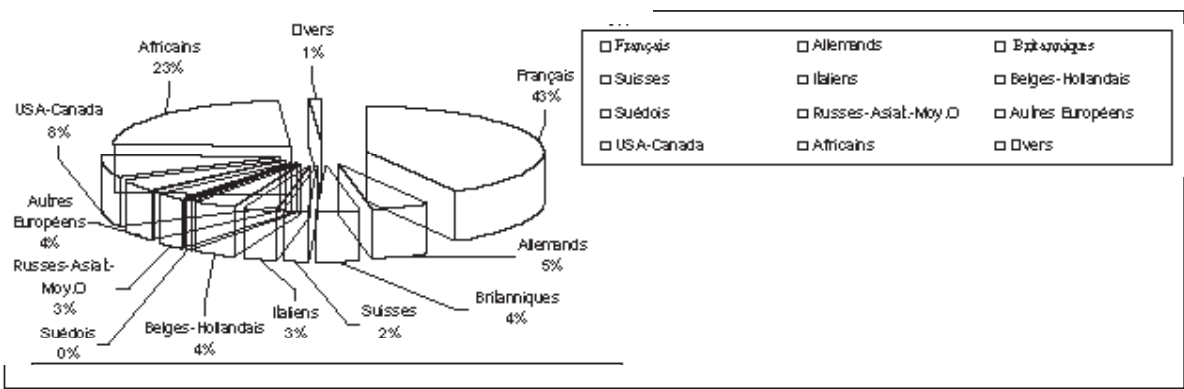

Fig.6b - Répartition des nuitées par nationalité (1976-1995) 
TCHINDJANG, M.; FODOUOP, K. Le Cameroun: un pays aux potentialites touristiques sous exploitees.

écourtement de la durée de séjour qui est inférieure à 2 jours en moyenne. En effet on constate une certaine évolution de la demande touristique de 1976 à 1986 caractérisée par une augmentation progressive et continue. Ensuite de 1987 à 1997, une baisse continuelle liée à la persistance des tensions politiques, la médiocrité des prestations et le vieillissement de certains établissements. Si l'activité est en déclin, les recettes aussi sont en baisse. Les quelques données glanées çà et là le prouvent bien. L'évolution indiciaire de la fréquentation touristique et taux d'accroissement montre une décroissance et apparaît par endroit en dents de scie. Toutes choses qui décrivent un malaise des visiteurs du Cameroun. Ce diagnostic semble décrire un système de gestion touristique bancale en dépit d'un certain assainissement du parc hôtelier qui montrent la gamme des hôtels au Cameroun par province et par lits. Enfin, si l'on regarde le budget en dollar US, on constate que le budget de l'Etat camerounais est stable ou évolue alors que celui du tourisme baisse (Fig. 7 a et b). Les raisons d'une telle situation sont multiples.
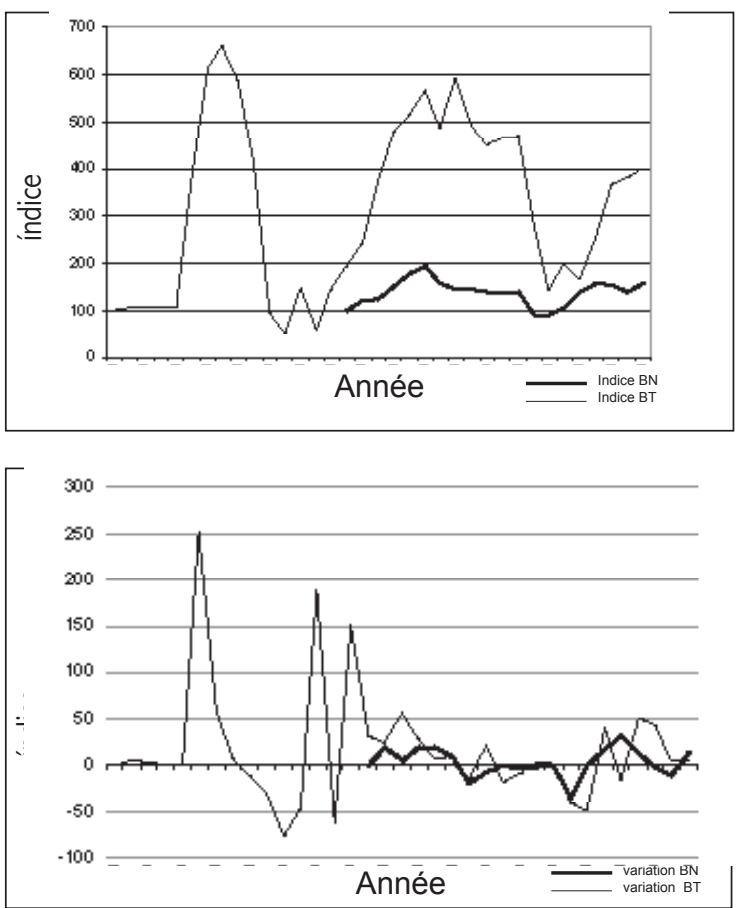

Fig. 7a - Evolution indiciaire $d u$ budget national et du budget du tourisme au Cameroun (1966-2001)

Fig. $7 b$ - Variation du budget national et de celui du tourisme (1966-2001) 
TCHINDJANG, M.; FODOUOP, K. Le Cameroun: un pays aux potentialites touristiques sous exploitees.

\section{Raisons du déclin et solutions}

\section{A - Raisons}

Les carences touristiques du Cameroun relèvent de plusieurs facteurs:

- Un réseau routier défectueux, inadapté ou vétuste, non entretenu à rénover ou à créer. Il existe au Cameroun très peu de routes bitumées. Les quelques donnée statistiques et les graphiques montrent jusqu'en $199462.131 \mathrm{~km}$ de routes avec $3.719 \mathrm{~km}$ de routes bitumées seulement ce qui fait une proportion de $5 \%$ (Fig. 8 a, b et c ). L'accès aux sites étant rendu difficile par la qualité des routes, leur valorisation touristique diminue en conséquence.
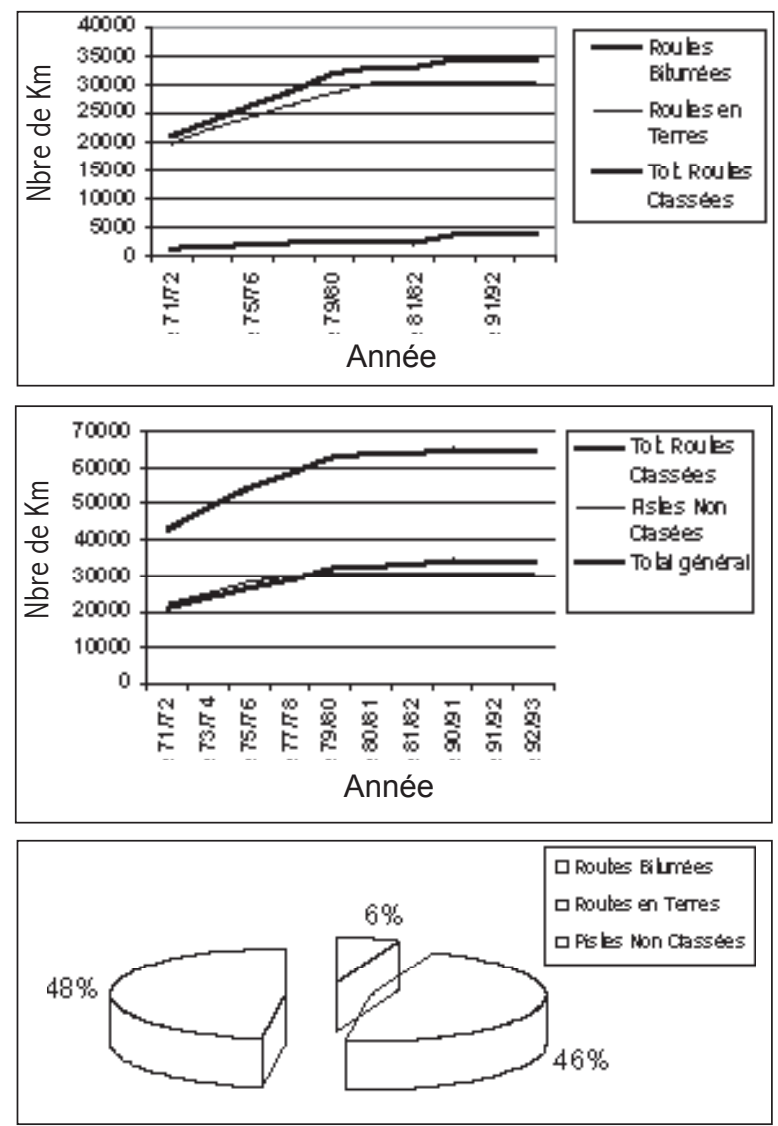

Fig.8a-Evolution du réseau routier au Cameroun depuis 1971

Fig. 8 b-Routes Classées et routes non classées depuis 1971

Fig. 8 c - Classification du réseau routier au Cameroun 
TCHINDJANG, M.; FODOUOP, K. Le Cameroun: un pays aux potentialites touristiques sous exploitees.

- La gestion inadéquate et l'insuffisance des outils statistiques découlant des observations ou des relevés. Ainsi, les données des fréquentations de certaines périodes sont perdues ou inexistantes et traduisent la légèreté avec laquelle les dites données sont relevées, conservées ou/et transmises aux services compétents. Les données des fréquentations touristiques sont en baisse suivant la courbe de variation qui constitue un indicateur fiable (Fig. 9 a, b et c).

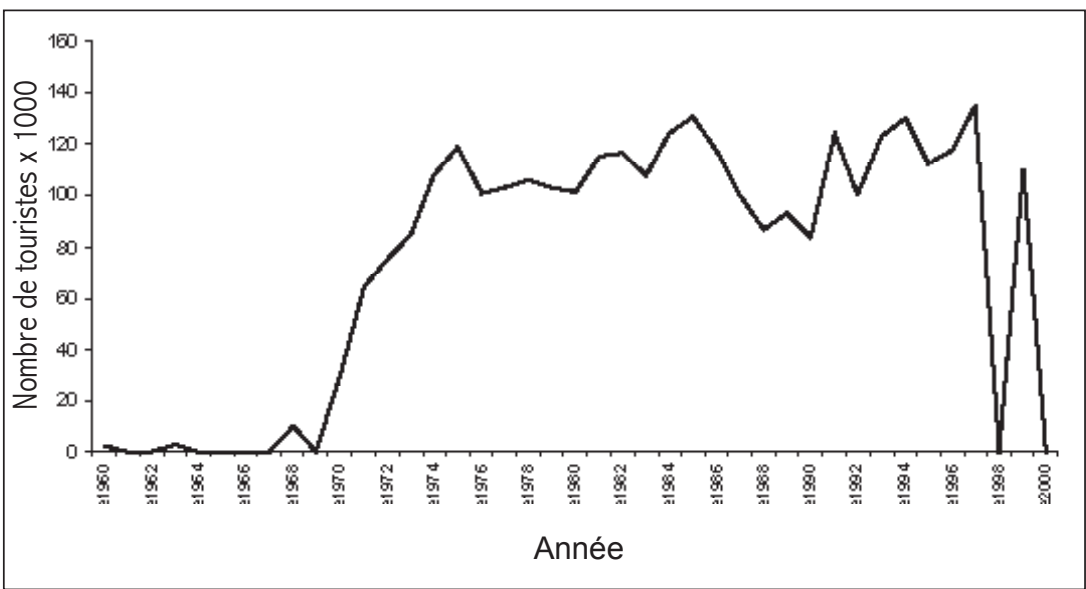

Fig.9a - Evolution de la fréquentation touristique du Cameroun de 1960 à 2000 (x 1000)

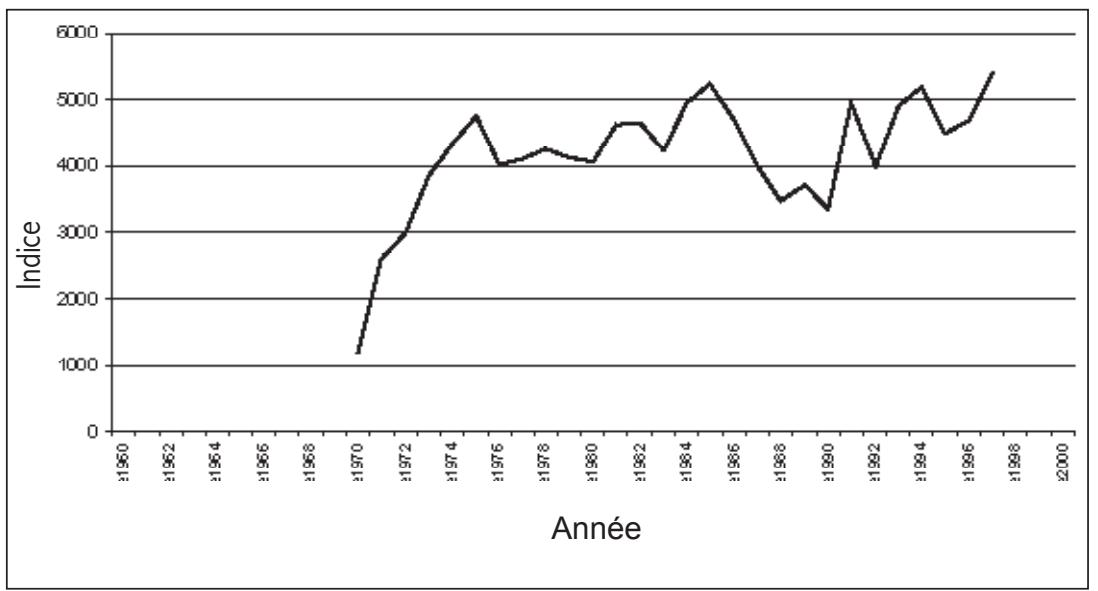

Fig.9b - Evolution indiciaire de la fréquentation touristique du Cameroun (1960-2000) 
TCHINDJANG, M.; FODOUOP, K. Le Cameroun: un pays aux potentialites touristiques sous exploitees.

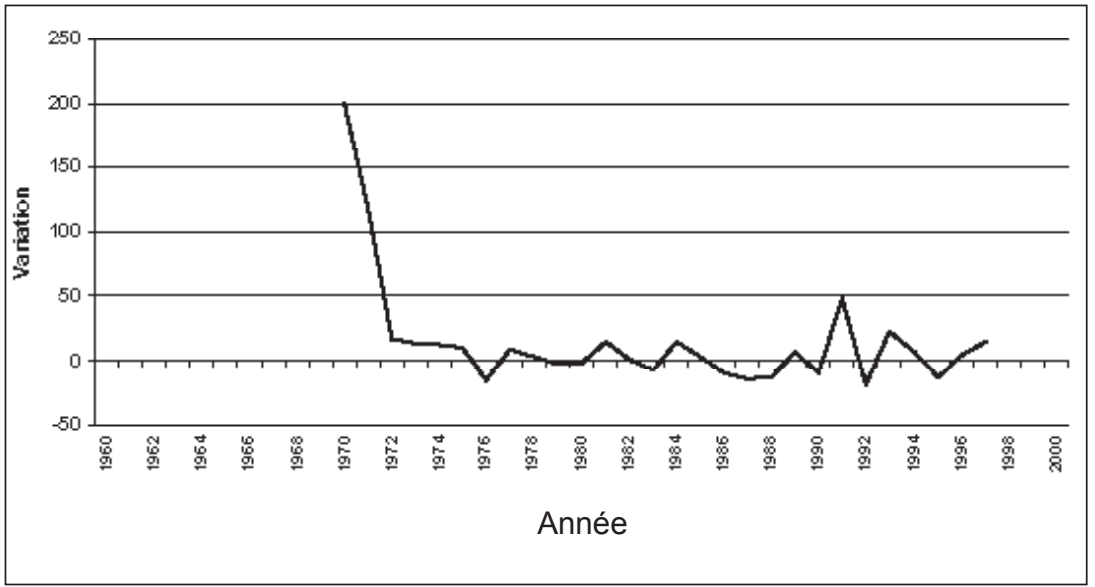

Fig.9c - Variation de la fréquention touristique au Cameroun de 1960 à 2000

- L'absence de cartographie et de zonation des sites. Par ailleurs, l'idée de site n'est pas bien ancrée dans les esprits. On se borne à perpétuer les sites classiques d'un tourisme naïf. Or dans la localisation touristique, le site est une entité naturelle spatiale composée de un ou de plusieurs éléments parmi lesquels le relief (montagne, colline, inselberg, versant), la végétation et la faune, les cours d'eau et les surfaces en eau; le climat et la pétrographie etc.

- La coopération avec le secteur privé est latente. En effet, le Gouvernement camerounais est trop regardant en matière de recettes et d'impôts, ce qui gène énormément les opérateurs privés engagés dans le tourisme. Dans ce sens, les données de fréquentations et de recettes sont parfois revues à la baisse. Des exemptions fiscales et des incitations monétaires devraient être des mesures d'accompagnement de la promotion touristique dans les zones défavorisées pour la multiplication des établissements d'hébergement et la décentralisation des 2 grandes métropoles du Cameroun.

- L'insécurité est l'une des causes de la chute de la fréquentation touristique. Celle-ci est un véritable désastre dans les pays africains. Ainsi au Zimbabwe, la fréquentation est passée de 1million de touriste en 1998 à 700.000 en 2000. Au Cameroun, l'insécurité tire ses origines de 3 événements majeurs. Dans un premier temps, elle provient des tensions sociales liées à la démocratisation; ces tensions ont provoqué une vague de violences de villes mortes dont le débouché 
TCHINDJANG, M.; FODOUOP, K. Le Cameroun: un pays aux potentialites touristiques sous exploitees.

logique a été dans la partie septentrionale du pays l'apparition des coupeurs de route. Ce phénomène a paralysé la demande touristique. L'insécurité dans un deuxième temps est liée à la délinquance et au grand banditisme provenant dans une certaine mesure de l'accroissement de la pauvreté depuis la dévaluation du franc CFA et les mesures d'ajustement structurel. L'insécurité est liée enfin à la misère grandissante qui envahit les villes et campagnes du pays et poussent certaines populations locales à des attitudes de mendicité pour les moins ambiguës, sinon agressives à l'endroit des touristes qui en sont gênés et freinés nets dans leur élan et soif de découverte. Ceci relève d'une approche non intégrée de la gestion touristique et du manque de sensibilisation (ou d'éducation et formation au tourisme): peu de guides locaux formés et instruits etc.

- La cherté de la destination camerounaise et les tracasseries policières (contrôles routiers fréquents, Visa d'entrée coûteux et le prix du billet d'avion qui ne facilite pas la proximité de l'Europe dont la clientèle est dominante par rapport au Kenya ou à l'Afrique du Sud qui est le premier pays touristique actuellement).

- L'absence d'une politique touristique fiable, convaincante et suffisamment attrayante; et, d'un plan directeur de Planification touristique réaliste et applicable à court et moyen terme. Le MINTOUR avait décidé à la création en 1989 d'élaborer un schéma directeur de promotion touristique! Mais on ne sait pas trop à quel niveau on se trouve ! Toujours est-il qu'il est bon de préciser qu'avec l'évolution mondiale actuelle, on ne peut guère imposer le tourisme aux populations sans les associer et sans trouver un moyen de réduire la pauvreté et d'encourager la formation des guides et l'alphabétisation des masses rurales défavorisées.

- L'insuffisance de la formation professionnelle en matière de tourisme. Ladite formation se limite aux métiers d'hôtellerie et ne met nullement l'accent sur l'inventaire des ressources naturelles et des potentialités touristiques du pays. De plus les mentalités des populations locales ne sont pas sensibilisées ou mieux converti vers la chose touristique. En effet, la pauvreté crée des situations de pressions sur les ressources engendrant le tourisme (braconnage, escroquerie et autres pratiques mafieuses). Il apparaît urgent de cultiver la notion de patrimoine dans les différentes tribus. Le patrimoine touristique étant ce que l'on hérite des générations précédentes et que l'on choisit de transmettre aux générations 

exploitees.

futures (sans pour autant les brader) dans un clan ou une tribu, un peuple ou une nation. Un patrimoine c'est ce qui fait l'identité ou la renommée d'un clan d'une tribu, ce qui donne une valeur, un titre au clan: un rocher, un totem, un palais, un oiseau, bref tout symbole investi par l'esprit du cal ou des ancêtres. Dans ce sens, le patrimoine est représentatif de la culture d'un peuple à une époque donnée et dès il constitue un potentiel touristique! Et Dieu seul sait que le Cameroun en regorge.

- L'insuffisance et le manque d'informations pour vanter la chose touristique à l'extérieur du pays et déclencher ainsi le flux. Les dépliants imprimés servent à autre chose qu'à à des expéditions et dépôts dans les Ambassades du Cameroun, les compagnies de transport ou les agences de voyage disséminées de par le monde. Par ailleurs, il n'existe plus de circuits touristiques au Cameroun à proprement parler en dehors de celui de l'écotourisme à Buéa dans la Province du Sud-Ouest. Par conséquent, sur le plan conceptuel, il y a léthargie et même absence de compétence. Enfin il manque des systèmes de campements mobiles à l'image de ce qui se fait au Kenya qui n'a misé pour son tourisme que sur ses aires protégées avec à son actif 1,05 millions de visiteurs en 1998 pour 400 millions de $\$$ de recettes.

- La faiblesse des investissements alloués à la promotion touristique. En effet, une analyse du Budget du MINTOUR montre que la plus grosse partie du Budget est consacrée au fonctionnement qui à la création prenait la totalité des fonds du ministère et dont le taux est passé de $100 \%$ à $65 \%$ alors que l'investissement est passé de $0 \%$ en 1990 à $35 \%$ en 2000. On comprend dès lors le piétinement du MINTOUR par rapport à la promotion touristique qui doit être une priorité absolue!

\section{B - Autres Recommandations}

En plus des propositions élaborées précédemment, il apparaît urgent au Cameroun de prendre quatre ordres de mesures concrètes :

- La mise au point des critères d'évaluation de la qualité et de la potentialité touristique des paysages sur le double plan quantitatif (fréquentation touristique) et qualitatif (consommation visuelle des paysages).

- La schématisation des paysages appuyés par l'observation. Ce volet est un préalable à un travail approfondi qui est la cartographie des paysages touristiques. Celle-ci est latente sinon absente en tout cas. 
TCHINDJANG, M.; FODOUOP, K. Le Cameroun: un pays aux potentialites touristiques sous exploitees.

- L'identification des acteurs de l'évolution des paysages et de la logique socioéconomique qui permet l'élaboration du maintien de la dynamique des paysages naturels. Le paysage étant selon Pitte une réalité culturelle résultant d'une combinaison du labeur humain sur la nature et de l'observation consommation. Le paysage touristique est un patrimoine qui se modifie si l'on ne s'en soucie pas; il décline et on l'anéantit. Par ailleurs tout paysage culturel a valeur de patrimoine et comme tel, il mérite d'être entretenu. Or, entretenir un paysage revient à le déchiffrer, lire son histoire et son évolution et au besoin l'archiver. Aussi, devons-nous retourner aux sources de la culture locale pour comprendre et déchiffrer le paysage au risque de le péricliter au nom du tourisme: c'est le cas de l'exploitation forestière effrénée ou du braconnage de la faune.

- La prise en compte réelle des cultures camerounaises et l'incitation aux résurgences des pratiques culturelles attrayantes qui sont en voie de disparition. La culture camerounaise est très riche, en effet avec 200 ethnies, le Cameroun est un véritable carrefour des traditions africaines allant des plus primitives (Pygmées et Mousgoum) aux civilisations traditionnelles les plus élaborées (civilisation Bamiléké et foulbé).

\section{Conclusion}

Certes, le Cameroun a accompli des efforts en matière d'assainissement de la gestion hôtelière (augmentation significative en qualité et en quantité du nombre d'hôtels) et de la privatisation. Mais beaucoup restent à faire notamment la suppression des visas pour le touriste au départ de l'étranger, la réduction des contrôles routiers et des tracasseries policières. Mais il y a également l'épineux problème de la chute du niveau de vie qui nécessite un plan de relance de la croissance économique, un plan de lutte contre la pauvreté en même temps qu'une sensibilisation à la notion de patrimoine par la mise en place d'une structure de dialogue et de concertation. Il faut également aux Camerounais, un minimum de volonté pour sortir ce pays, véritable scandale géographique (en comparaison toutes proportions gardées à l'Afrique du Sud et au Brésil) et paradoxe touristique de cette léthargie et de ce sommeil. Ceci s'accompagnera d'un inventaire critique et objectif du site patrimoine et du poids des facteurs géographiques dans la localisation touristique.

Remerciements: Nos remerciements à Mr MBOHOU Souleymanou, 
TCHINDJANG, M.; FODOUOP, K. Le Cameroun: un pays aux potentialites touristiques sous exploitees.

Directeur des Normes et Procédures au MINTOUR, qui a bien voulu lire le manuscrit et pour ses précieux conseils dans la réalisation de ce travail ainsi qu'à son collègue MAHEND Elisée pour les statistiques sur la Loi des Finances du Cameroun.

\section{RESUMO \\ Camarões: um país de potenciais turísticos subexplorados}

Possuindo uma vasta extensão em latitude $\left(11^{\circ}\right.$, Fig. 1a), um relevo variado constituído de planícies, platôs e montanhas, além de um clima e uma vegetação tão diversificados quanto sua população heterogênea, Camarões aparece como uma terra de turismo por excelência. É reconhecido por especialistas como uma África em miniatura. Entretanto, até o presente, o país não tem aproveitado esse rico potencial natural. Em efeito, a contribuição da atividade turística para o PIB de Camarões é insignificante (somente 1\%). A falta de instrumentos estatísticos confiáveis e a ausência de política de incitação tornam difícil qualquer iniciativa de avaliação dos recursos e das potencialidades turísticas do país. Para dizer a verdade, a atividade turística de Camarões está em declínio, como apontam as raras estatísticas sobre as freqüências e receitas aí relacionadas.

Unitermos: África em miniatura, cultura, pontos turísticos, turismo.

\section{BIBLIOGRAPHIE}

AFRIQUE, Magazine. Cameroun, terre de tourismes. Découverte, Afrique Magazine, Paris, n. 184, p. 71-85, 2001.

BARRETT, W; GEOFFREY; LEE, P. Gabriel. European community support for tourism in ACP states and regions: implications for the public and private sector. In: Privitisation in Tourism Conference, 15 nov. 1993, London. 13 p.

BRUNET, L. Pays en voie de développement et tourisme international. Bulletin de l'Association des Géographes Français, Paris, n. 377/378, p. 15-30, 1970.

EDWARDS, John. The privitisation process: some key legal issues. In: Privitisation in Tourism Conference, 15 nov. 1993, London. 10 p. 
TCHINDJANG, M.; FODOUOP, K. Le Cameroun: un pays aux potentialites touristiques sous exploitees.

L'INDUSTRIE du tourisme face au défi intégriste. L'Expression, Paris, n. 504, 1995.

EVERITT, Richard. Privitisation and tourism. In: Privitisation in Tourism Conference, 15 nov. 1993, London. 8 p.

FABRE, Pierre. Tourisme international et projets touristiques dans les pays en développement: méthodologie de la planification. Cameroun: Yaoundé, 1990. 420 p. Rapport MINTOUR.

HERBICH, Maciej. Investment opportunities in Poland's tourist industry. In: Privitisation in Tourism Conference, 15 nov. 1993. London. 6 p.

IMAGES Economiques du Monde. Paris: SEDES, 2001. 384 p.

CAMEROUN, cap sur l'an 2000: le tourisme. Jeune Afrique Economie, Paris, Edition spéciale hors série, p. 311-328, 1996.

LOZATO GIOTART, Jean Pierre. Géographie du Tourisme: de l'espace regardé à 1'espace consommé. $3^{\text {ème }}$ édition. Paris, Barcelone, Milan: Masson, 1989. 309 p.

MAINET, Nicole. Les aspects géographiques du tourisme au Cameroun. Thèse $3^{\text {ème }}$ cycle, Université de Bordeaux III. Bordeaux, 1979. 268 p., 77 fig., 13 planches.

MAINET, Nicole. Tourisme et montagne en Afrique. Actes du Colloque SEPANRIT L4 home et la Montagne Tropicale, Yaoundé, p. 147-150, 31 mars-13 avril 1983. Bordeaux, 1988.

MINISTÈRE DE LA. Tourisme international et projet touristique dans les pays en voie de développement. Paris, 1979. 274 p.

MINTOUR. La nouvelle politique touristique du Cameroun. Yaoundé, 1994. $50 \mathrm{p}$.

MINTOUR. La nouvelle politique touristique du Cameroun: document de synthèse. Yaoundé, 1996. 17 p.

MORUCCI, Bernard. Analyse comparative de politiques touristiques dans les pays industrialisés et dans les pays en voie de développement. Colloque TEOROS. Montréal: Université du Québec. p.1-12, 21-24 mai 1991.

MOSYOKA, E. M. Privitisation: the Kenyan case. In: Privitisation in Tourism 
TCHINDJANG, M.; FODOUOP, K. Le Cameroun: un pays aux potentialites touristiques sous exploitees.

Conference, 15 nov. 1993, London. 13p.

OMT. Mission d'appui sectoriel au Cameroun sur les besoins pour la transformation de l'ENAHT en centre sous régional dans le domaine de l'hôtellerie et du tourisme. Yaoundé, 1992. 12 p. Rapport pour le Cameroun par François DUVERGE.

STEIGENBERGER CONSULTING GMBH. Planification touristique au Cameroun: analyse des principaux pays concurrentiels du Cameroun: Sénégal, Sierra Leone, Liberia, Côte d'Ivoire, Ghana, Togo, Dahomey (Bénin), Nigeria, Gabon, Kenya. Francfort/Main, 1973. 158 p.

STEIGENBERGER CONSULTING GMBH. Rentabilité en devises des investissements touristiques au Cameroun. Francfort/Main, 1976. 106 p.

Recebido em: 8/8/2003 